\title{
Beckmann rearrangement of acetophenone oximes to the corresponding amides organo-catalyzed by trifluoroacetic acid for sustainable NSAIDs synthesis
}

\author{
Giuseppe Quartarone, Elia Rancan, Lucio Ronchin*, Andrea Vavasori \\ Department of Molecular Sciences and Nanosystems, University Ca' Foscari of Venice, Dorsoduro 2137, 30123, Venezia, Italy
}

\section{A R T I C L E I N F O}

\section{Article history:}

Received 16 September 2013

Received in revised form

27 November 2013

Accepted 21 December 2013

Available online 28 December 2013

\section{Keywords:}

Trifluoroacetic acid

Organocatalysis

Beckmann rearrangement

Acetaminophen

\begin{abstract}
A B S T R A C T
The Beckmann rearrangement of acetophenone oximes to the corresponding amides (4hydroxyacetophenone oxime to $\mathrm{N}$-acetyl-4-hydroxyacetanilide and acetophenone oxime to $\mathrm{N}$-phenylacetamide) is investigated by using trifluoroacetic acid (TFA) as catalyst. The reaction occurs either in the presence or in the absence of a suitable solvent. High selectivity and practically quantitative yield to amide is achieved in both cases at TFA/substrate $>3$. Both TFA and the solvent (whenever present) could be easily reused by distillation since no protonation of amides occurs. The reaction proceeds via a multistep reaction path and the role of TFA is related not only to its acidity but also mainly to its ability on forming reactive trifluoroacetylated intermediates. In particular, the highly reactive trifluoroacetylated amide is actually the effective catalyst. Finally, a likely reaction path is proposed.
\end{abstract}

(c) 2014 Elsevier B.V. All rights reserved.

\section{Introduction}

Acetanilide (N-phenylacetamide) and paracetamol or acetaminophen (N-acetyl-4-aminophenol) are antipyreticanalgesic drugs. Besides acetanilide has been employed in drug formulations for long time, though, now for this use it is almost completely substituted by acetaminophen [1-5]. In any case, acetanilide is an important intermediate in the production of fine chemicals, in fact, it is used as an inhibitor of peroxides, stabilizer for cellulose ester varnishes, as an intermediate for the synthesis of rubber accelerators, dye intermediate and as a precursor in penicillin synthesis [5].

Acetaminophen production is in continuous growth and its industrial synthesis is based on four different routes: (i) from phenol, (ii) from chlorobenzene, (iii) from nitrobenzene and (iv) from 4-hydroxyacetophenone oxime (4-HAPO), the latter known also as the Hoechst-Celanese process [2,3]. In the past, the first two processes were predominant, while the last two have been evolving in recent years [3]. From economical and environmental point of view the first two show more concerns, due to the large coproduction of unwanted byproducts (such as isomers and salts). In addition, the processes are carried out in batch reactors, thus implying small production with high costs.

\footnotetext{
* Corresponding author.

E-mail address: ronchin@unive.it (L. Ronchin).
}

Conversely, both the route via selective nitrobenzene hydrogenation to 4-aminophenol and the Hoechst-Celanese process are continuous multistage plant developed for large productions [1-4]. These processes involve the use of acid catalysts and the plants in operation are actually using mineral acids, which limit their sustainability from both environmental and economical point of view [4]. The latter, particularly, comprises several stages where acid catalysts are involved: the Fries rearrangement of phenyl acetate, the oximation of the 4-hydroxyacetophenone to 4-HAPO and finally its Beckmann rearrangement to paracetamol [1-3]. Above all, Fries and Beckmann rearrangement require strong acids and severe reaction conditions [1-3]. Both the stages employ mineral acid and inorganic bases each one with the necessary hold-up operation, clearly their sustainability improvement passes through the use of easily reusable acid catalytic system.

The studies related to the Beckmann rearrangement have been particularly focused on the cyclohexanone oxime to $\varepsilon$-caprolactam conversion, since this reaction is of paramount importance for the industry, being this lactam the key intermediate for nylon 6 production [6-10]. A large number of papers dealing to the Beckmann rearrangement of ketoximes are available in literature employing liquid and solid acid catalysts (both inorganic and organic) [11-18], but few papers are specifically available on the Beckmann rearrangement of the 4-hydroxy acetophenone oxime as intermediate for acetaminophen synthesis [14-17]. In patent literature the most important application are referred to the Celanese process but there are some patents claiming the Beckmann rearrangement of ketoximes to the corresponding amides $[2,3,19,20]$. 


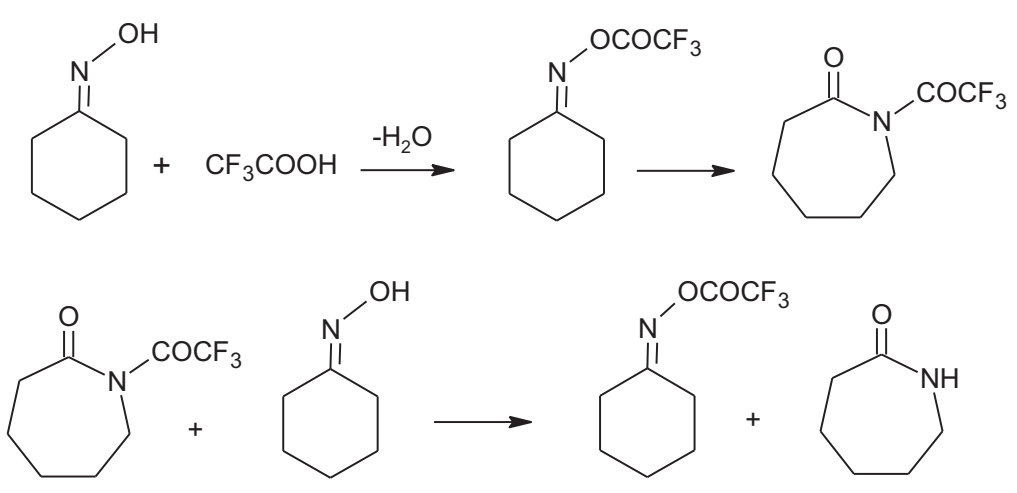

Scheme 1. Schematic reaction mechanism of TFA catalyzed Beckmann rearrangement of cyclohexanone oxime to $\varepsilon$-caprolactam see Ref. [28].

Among the liquid acids TFA appears to be really interesting for synthetic purposes, because of its low boiling point (346 K), which allows an easy recovery and recycling of the acid by distillation without products decomposition $[21,22]$. In addition, TFA is non toxic, quite stable, its decomposition does not give harmful products and it does not give accumulation phenomena in the environment [23]. However, except the paper of Cossy and coworkers, who employed TFA for the rearrangement of ketoxime carbonate [24], TFA was not used for this reaction as a catalyst and only in few cases it was employed in the solvent system but not recognized as catalyst [25]. During the last 5 years our research group has studied the Beckmann rearrangement of cyclohexanone oxime to $\varepsilon$-caprolactam in the presence of TFA as catalyst [26-29]. For instance, we showed that TFA in $\mathrm{CH}_{3} \mathrm{CN}$ as a solvent gives practically quantitative conversion with high selectivity in the Beckmann rearrangement of cyclohexanone oxime to $\varepsilon$ caprolactam [27-29]. Furthermore, the solvent catalytic system $\mathrm{CH}_{3} \mathrm{CN}$-TFA is fully reusable because of the acidity of the TFA does not allow the formation of the caprolactam salt, typically observed with mineral acids, thus it does not need neutralization [26-29]. Those papers clearly evidenced that the reaction occurs via an organocatalyzed path due to the formation of compounds involving TFA esters [26-29]. The proposed reaction mechanism envisages the formation of the oxime ester of the trifluoroacetic acid, which, after rearrangement, forms a trifluoroacetyl acetamide. The latter is the key intermediate of the catalysis being the trifluoroacetylating compound of the oxime, which continuously reforms the ester sustaining the catalytic cycle (Scheme 1).

Very recently Luo and co-workers deposited a patent for the synthesis of amides, based on the use of TFA- $\mathrm{CH}_{3} \mathrm{CN}$ system [30]. As a matter of fact, the application claims a procedure quite similar to that proposed in our previous papers and the final results are practically overlapping [26-30].

In this work we study TFA as catalyst for the Beckmann rearrangement of aromatic ketoximes to the corresponding amides, employing both APO and 4-HAPO as substrates and we show that TFA acts in both cases as an organocatalyst. In addition, we investigate the influence of different solvents (also neat TFA) and of the operative variables on reaction rate conversion and selectivity. Finally, on the basis of the reactivity of the intermediates we propose a reaction mechanism, which explains the behaviors of both the oximes.

\section{Experimental}

\subsection{Materials}

All solvents and products were employed as received without further purification. Acetophenone $\geq 98 \%$, trifluoroacetic acid $99 \%$, trifluoroacetic anhydride $>99 \%$, nitroethane $96 \%$ and hydroxylamine hydrochloride 99\%, dimethyl carbonate were Aldrich products; 4-hydroxiacethophenone $\geq 98 \%$ (HPLC), dichloromethane, acetonitrile, nitroethane, were all HPLC grade Fluka products. Dimethyl sulfoxide (DMSO) 99\% and 1,2-dichloroethane 99\% were ACS reagent Aldrich. Deuterated chloroform, deuterium oxide and deuterated dimethyl sulfoxide $\left(\right.$ DMSO- $\left.d_{6}\right)$ were EurisoTop products.

\subsection{Instruments}

The gas-chromatograph (GC) Agilent model 6890, equipped with a HP5, film thickness $2.65 \mu \mathrm{m}, 30 \mathrm{~m} \times 530 \mu \mathrm{m}$ column; detector: flame ionization detector (FID); carrier gas: $\mathrm{N}_{2}, 0.9 \mathrm{~mL} / \mathrm{min}$; oven: $333-523 \mathrm{~K}(5 \mathrm{~min})$ at $5 \mathrm{~K} / \mathrm{min}$ was used mainly for the analysis of APO reactions.

The quantitative analyses of 4-HAPO rearrangement were carried out exclusively by high performance liquid chromatography (HPLC), because 4-HAPO and acetaminophen decompose into the GC injector. The HPLC instrument employed was a Perkin Elmer binary LC pump 250 with phenomenex Luna, $5 \mu \mathrm{m} \mathrm{C18} 100 \AA$, LC column $300 \mathrm{~mm} \times 4.6 \mathrm{~mm}$ (detector: Perkin Elmer LC $235 \mathrm{C}$ Diode Array, wavelengths: $255 \mathrm{~nm}$ and $220 \mathrm{~nm}$; carrier: mix water - acetonitrile with a concentration gradient $60 \%$ water $(9 \mathrm{~min}), 50 \%$ water ( $5 \mathrm{~min}$ ) and $30 \%$ water (1 $\mathrm{min})$ ).

Despite of both APO and acetanilide are stable by GC analysis the rearrangement was followed by using either GC or HPLC, since intermediates appears to decompose by using both the techniques.

The gas-chromatograph coupled mass spectroscopy (GC-MS) Agilent model 5975C interfaced with GC Agilent model 7890 equipped with a HP5 film thickness $0.5 \mu \mathrm{m}, 30 \mathrm{~m} \times 250 \mu \mathrm{m}$ was used throughout the experiments for checking reaction products. The reaction carried out in the presence of $\mathrm{H}_{2} \mathrm{SO}_{4}$ and $\mathrm{CH}_{3} \mathrm{SO}_{3} \mathrm{H}$ were analyzed after dilution with water and extraction with dichloromethane, the rest were analyzed without any neutralization.

The ${ }^{1} \mathrm{H}$ NMR spectra were recorded on a Bruker AC 200 spectrometer operating at $200.13 \mathrm{MHz}$, the sample temperature was maintained at $298 \mathrm{~K}$. The reagent concentrations were chosen in order to simulate the real catalytic conditions. All the chemical shifts were referred to internal tetramethylsilane.

\subsection{Ketoxime synthesis}

4-HAPO and APO were synthesized from the corresponding ketone (4-hydroxyacetophenone and acetophenone) by following the procedure described in literature $[27,28]$. In a typical preparation, a flask equipment equipped with reflux condenser was charged with $170 \mathrm{~mL}$ of water, $0.51 \mathrm{~mol}$ of hydroxylamine hydrochloride, $0.81 \mathrm{~mol}$ of sodium acetate and $0.15 \mathrm{~mol}$ of 4 -HAPO. The reaction was heated at $c a .363 \mathrm{~K}$ for $1 \mathrm{~h}$ and then, after cooling, 
the solid was filter and washed with a saturated solution of $\mathrm{NaCl}$ and $\mathrm{NaHCO}_{3}$ at $273 \mathrm{~K}$. The crude 4-HAPO was dissolved in water and extracted with diethyl ether. After evaporation of the solvent the solid was twice recrystallized with diethyl ether and hexane. NMR assignments:

4-HAPO, white solid, m.p. 413-418 $\mathrm{K},{ }^{1} \mathrm{H}$ NMR: (200 MHz, $\left.\left(\mathrm{CD}_{3}\right)_{2} \mathrm{SO}\right), \delta 10.84(\mathrm{~s}, 1 \mathrm{H}, \mathrm{N}-\mathrm{OH}), 9.60(\mathrm{~s}, 1 \mathrm{H},-\mathrm{OH}), 7.49-7.45(\mathrm{~d}$, $2 \mathrm{H}), 6.78-6.73(\mathrm{~d}, 2 \mathrm{H}), 2.09(\mathrm{~s}, 3 \mathrm{H})[31]$;

APO, white solid, m.p. 331-333 K, ${ }^{1} \mathrm{H}$ NMR: $\left(200 \mathrm{MHz}, \mathrm{CDCl}_{3}\right), \delta$ 9.04 (br s, $1 \mathrm{H},-\mathrm{OH}), 7.68-7.63(\mathrm{~m}, 2 \mathrm{H}), 7.43-7.40$ (m, 3H), 2.34 (s, $3 \mathrm{H})[32]$.

\subsection{Synthesis of O-trifluoroacetyl oximes ester, $N$-trifluoroacetyl acetanilide, $\mathrm{N}$-trifluoroacetyl 4-hydroxyacetanilide}

An attempt to obtain O-trifluoroacetyl-4-hydroxyacetophenone oxime (4-HAPO-TFA) by following the procedure reported for cyclohexanone oxime in previous papers does not give satisfactorily results $[27,28]$. In fact, the formation of 4-HAPO-TFA by reacting trifluoroacetic anhydride with 4-HAPO is not observed since, the instantaneous rearrangement to acetaminophen is observed also at $298 \mathrm{~K}$.

The reactivity of trifluoroacetic anhydride with acetophenone oxime at $353 \mathrm{~K}$ gives in few minutes acetanilide but, O-trifluoroacetyl acetophenone oxime (APO-TFA) is formed at $298 \mathrm{~K}$ by following the procedure reported for cyclohexanone oxime in previous papers $[27,28]$. The reaction occurs almost quantitatively in various solvent in a typical preparation $25 \mathrm{mmol}$ of APO reacts with an equimolar amount of trifluoroacetic anhydride in $3 \mathrm{~mL}$ of $\mathrm{CH}_{2} \mathrm{Cl}_{2}$. To avoid fast decomposition of the products, APO-TFA is maintained in a DMSO solution in the presence of an over-stoichiometric amount of trifluoroacetic anhydride (1.1 equiv. with respect to APO-TFA). The product is identified by ${ }^{1} \mathrm{H}$ NMR, GC-MS and HPLC (see supplementary materials).

$\mathrm{N}$-Trifluoroacetyl-4-hydroxyacetanilide (AcP-TFA) or $\mathrm{N}$-trifluoroacetyl-acetanilide (AcA-TFA) are obtained by trifluoroacetylation of acetaminophen or acetanilide in acetonitrile, the products are identified via GC-MS and NMR (see supplementary materials). In a typical preparation $26 \mathrm{mmol}$ of paracetamol was dissolved with $3 \mathrm{~mL}$ of acetonitrile and added $26 \mathrm{mmol}$ of trifluoroacetic anhydride in a glass flask, then the reaction was stirred for $1 \mathrm{~h}$ at $298 \mathrm{~K}$. The solvent was eliminated under vacuum by a rotary evaporator at $298 \mathrm{~K}$; a deliquescent white solid was obtained and analyzed by HPLC, GC-MS and NMR (see supplementary materials). Analogous procedure was followed to prepare $\mathrm{N}$-trifluoroacetyl acetanilide (see supplementary materials). It is noteworthy that all the compounds are moisture sensitive. To avoid fast decomposition of the products, both AcP-TFA and AcA-TFA are maintained in a DMSO or $\mathrm{CH}_{3} \mathrm{CN}$ solution in the presence of an over-stoichiometric amount of TFA (1.1 equiv. with respect to AcP-TFA or AcA-TFA).

NMR assignments:

APO-TFA, ${ }^{1} \mathrm{H}$ NMR (200 MHz, $\left.\mathrm{CDCl}_{3}\right), \delta 7.75-7.70(\mathrm{~m}, 2 \mathrm{H})$, 7.54-7.26 (m, 3H), $2.51(\mathrm{~s}, 3 \mathrm{H})$;

acetanilide, white solid m.p. $385-389 \mathrm{~K},{ }^{1} \mathrm{H} \mathrm{NMR}\left(200 \mathrm{MHz}, \mathrm{CDCl}_{3}\right)$, $\delta 8.19$ (br s, $1 \mathrm{NH}), 7.55-7.51(\mathrm{~m}, 2 \mathrm{H}), 7.33-7.25(\mathrm{~m}, 2 \mathrm{H}), 7.13-7.06$ (m, 1H), $2.15(\mathrm{~s}, 3 \mathrm{H})[33]$;

AcA-TFA, ${ }^{1} \mathrm{H}$ NMR $\left(200 \mathrm{MHz}, \mathrm{CDCl}_{3}\right), \delta 7.52-7.47(\mathrm{~m}, 3 \mathrm{H})$, 7.25-7.20 (m, 2H), $2.55(\mathrm{~s}, 3 \mathrm{H})$;

acetaminophen, white solid m.p. $440-443 \mathrm{~K}{ }^{1} \mathrm{H}$ NMR $\left(\left(\mathrm{CD}_{3}\right)_{2} \mathrm{SO}\right.$, $200 \mathrm{MHz}$ ), $\delta 9.63$ (s, 1H, $-\mathrm{OH}$ ), 9.12 (br s, $1 \mathrm{H},-\mathrm{NH}), 7.36-7.31$ (d, $2 \mathrm{H}), 6.69-6.65$ (d, 2H), $1.98(\mathrm{~s}, 3 \mathrm{H})[34]$;
AcP-TFA, ${ }^{1} \mathrm{H}$ NMR $\delta 9.64$ (s, 1H, -OH), 7.45-7.32 (d, 2H), 6.70-6.65 (d, 2H), 1.97 (s, 3H).

\subsection{Beckmann rearrangement reactions}

All the operations were carried out under nitrogen (charged at atmospheric pressure) in a jacketed sealed glass reactor $(10 \mathrm{~mL})$ at several temperatures and autogenous pressure. The course of reactions was checked sampling the liquid phase by a syringe at established intervals. All the analyses were carried out by GC and GC-MS when APO was employed as substrate, while HPLC and GC-MS were used when the substrate was 4-HAPO. The potential of the method as an alternative synthetic route for obtaining different amides has been tested on several oximes giving, in any case, high yield in the corresponding amides (see supplementary materials).

In a typical experiment, a glass reactor was charged with $1.3 \mathrm{mmol}$ of 4 -HAPO, $9 \mathrm{~mL}$ of nitroethane and $12.5 \mathrm{mmol}$ of TFA under nitrogen. The reaction time was computed after the TFA addition.

The first derivative at time 0 of the third order polynomial, obtained by fitting the time decreasing concentration of 4-HAPO, gives the overall initial reaction rate, thus allowing the control of the influence of the operative variable on the reaction kinetics. In this case substrate consumption and amide formation has been followed by HPLC analysis since GC cannot be employed since either 4-HAPO or acetaminophen decompose in the GC injection system, giving not reliable results in the quantitative analysis.

The initial rate of APO rearrangement was measured by the first derivative at time 0 of the third order polynomial obtained by fitting the time $v s$. acetanilide formation data. The different methods in the calculation of the initial rate of reaction for the two substrate is due the fact that APO shows a complex reaction path, then acetanilide formation appears to be a simple and reproducible parameter for measuring the overall reaction rate.

\subsection{Products, solvent and catalyst recovery}

The reacted mixture is transferred to a mini distillation apparatus equipped with membrane pump, operating at $130 \mathrm{~Pa}$ and with a condenser chilled at $273 \mathrm{~K}$ with a circulating thermostat. The mixture is heated at $353 \mathrm{~K}$, than the solvent quickly distils. The solvent recovered is over $95 \%$ of the initial solvent, the rest is in the distillation apparatus. The residue is recovered and the isolated yield of acetanilide and acetaminophen are ca. 90-95\%.

\section{Results and discussion}

\subsection{Influence of the solvent on the Beckmann rearrangement of 4-HAPO and APO catalyzed by TFA}

Table 1 reports the influence of the solvent on the Beckmann rearrangement reaction of 4-HAPO and APO after $2 \mathrm{~h}$ of reaction.

It is noteworthy that conversions are close to completeness in the presence of various solvents, in some cases the reaction is practically quantitative to the desired amide, except entries 4, 6 and 8 that are carried out in the presence of ethanol, DMC and DMSO, respectively. As regard the reactivity in ethanol, the behavior is expected, since TFA reacts almost instantly with the alcohol to give ethyl trifluoroacetate, thus subtracting the acid to the reaction environment. In the presence of DMC the reaction does not proceed but no byproducts were observed. This strong solvent effect is generally related to a stabilization of a charged intermediate, whose stability increases the activation energy of the overall process [35]. This strong solvent interaction inhibits also all the reactions involved in the process (oxime hydrolysis and/or 
Table 1

Beckmann rearrangement of 4-HAPO and APO catalyzed by TFA in the presence of various solvent.

\begin{tabular}{|c|c|c|c|c|c|c|c|}
\hline \multirow[t]{2}{*}{ Entry } & \multirow[t]{2}{*}{ Solvent } & \multicolumn{3}{|l|}{ 4-HAPO } & \multicolumn{3}{|l|}{$\mathrm{APO}$} \\
\hline & & Conv. ${ }^{\mathrm{a}}(\%)$ & Select. $^{\mathrm{b}}(\%)$ & $T(\mathrm{~K})$ & Conv. ${ }^{\mathrm{a}}(\%)$ & Select. $^{\mathrm{c}}(\%)$ & $T(\mathrm{~K})$ \\
\hline 1 & Acetonitrile & 99 & 99 & 343 & $>99$ & $60.2^{\mathrm{d}}$ & 358 \\
\hline 2 & Nitromethane & $>99$ & 99 & 343 & $>99$ & 99 & 358 \\
\hline 3 & Nitroethane & $>99$ & $>99$ & 343 & $>99$ & $>99$ & 358 \\
\hline 4 & Ethanol & $p^{e}$ & 1 & 343 & $\rho^{e}$ & 1 & 358 \\
\hline 5 & 1,2-Dichloroethane & 97 & 96 & 343 & 96 & 95 & 358 \\
\hline 6 & DMC & $<1$ & 1 & 343 & $<1$ & 1 & 358 \\
\hline 7 & DMSO & 1 & l & 343 & 1 & l & 358 \\
\hline 8 & Chloroform & 96 & 96 & 343 & 96 & 98 & 358 \\
\hline 9 & TFA & 97 & 96 & 343 & $>99$ & 99 & 358 \\
\hline 10 & $\mathrm{CH}_{3} \mathrm{SO}_{3} \mathrm{H}$ & 98 & 99 & 343 & 99 & 99 & 358 \\
\hline 11 & $\mathrm{H}_{2} \mathrm{SO}_{4} 75 \%$ & 99 & 99 & 343 & 99 & 99 & 358 \\
\hline
\end{tabular}

Run condition: molar ratio $\mathrm{TFA} / \mathrm{substrate}=10, \mathrm{TFA}=13.2 \mathrm{mmol}$, total volume $=10.8 \mathrm{~mL}$, time of reaction $120 \mathrm{~min}$.

a Oxime conversion.

b Selectivity to acetaminophen.

c Selectivity to acetanilide.

d Formation of APO-TFA are observed; after $18 \mathrm{~h}$ of reaction acetanilide yield $99 \%$.

e TFA is converted quantitatively to its ethyl ester and reaction does not proceed further.

esterification). In fact, these reactions are observed for APO (entry 1 ) as well as for cyclohexanone oxime rearrangement in TFA- $\mathrm{CH}_{3} \mathrm{CN}$ [26-29]. Correspondingly, in DMSO (entry 7), the reaction does not proceed, likely for a similar solvent reason [35]. Entry 8 shows high conversion and selectivity for both 4-HAPO and APO in chloroform as the solvent.

Reactions carried out in neat TFA (entry 9) show high conversion and selectivity to the desired amide for either 4-HAPO or APO.

Entries 10 and 11 show the reactivity in $\mathrm{CH}_{3} \mathrm{SO}_{3} \mathrm{H}$ and $\mathrm{H}_{2} \mathrm{SO}_{4}$ $70 \%$, as expected in both cases high conversion and selectivity are achieved but dilution in water and extraction with dichloromethane needs to recover the products.

The procedure has also been tested on several ketoximes and aldoximes giving high yield in the desired amides, some preliminary results are available on supplementary materials but this is beyond the scope of the present research and further investigations are in course.

\subsection{Time vs. concentrations profile of the TFA catalyzed}

Beckmann rearrangement in nitroethane as a solvent

Fig. 1 shows the time $v s$. concentrations profile of the Beckmann rearrangement of 4-HAPO and APO catalyzed by TFA in nitroethane as a solvent.
The comparison of the two plots of Fig. 1 shows apparently two completely different trends. The 4-HAPO rearrangement shows the disappearing of the oxime with the concomitant increase of the relative amide (acetaminophen). On the contrary, the rearrangement of APO shows a complex reaction pattern, in which is evidenced that part of the starting oxime is transformed almost immediately in various intermediates after the addition of the TFA. Furthermore, acetanilide formation presents an induction period; this suggests that the formation of a catalytically active intermediate requires a certain time to reach the minimum concentration for starting the catalysis of the rearrangement to acetanilide. Such an induction period is temperature dependant (see supplementary materials) and decreases as temperature raises. The intermediate of Fig. 1(b) has been identified (GC-MS, NMR and compared with a standard see supplementary materials) as APO-TFA and AcA-TFA, while acetophenone derive from APO hydrolysis due to the reaction of the protonated form of APO (APO $\mathrm{p} K_{\mathrm{a}}=2.52$, TFA $\left.\mathrm{p} K_{\mathrm{a}} \approx 0.47\right)$ with the water deriving from the formation of the APO-TFA (esterification), or from a secondary reaction path already described in a previous paper [27-29,36-38].

This class of products, suggests a reaction path for the APO Beckmann rearrangement, similar to that proposed for cyclohexanone oxime $[27,28]$. On the contrary, the time $v s$. concentration profile of the 4-HAPO rearrangement would suggest a Brønsted
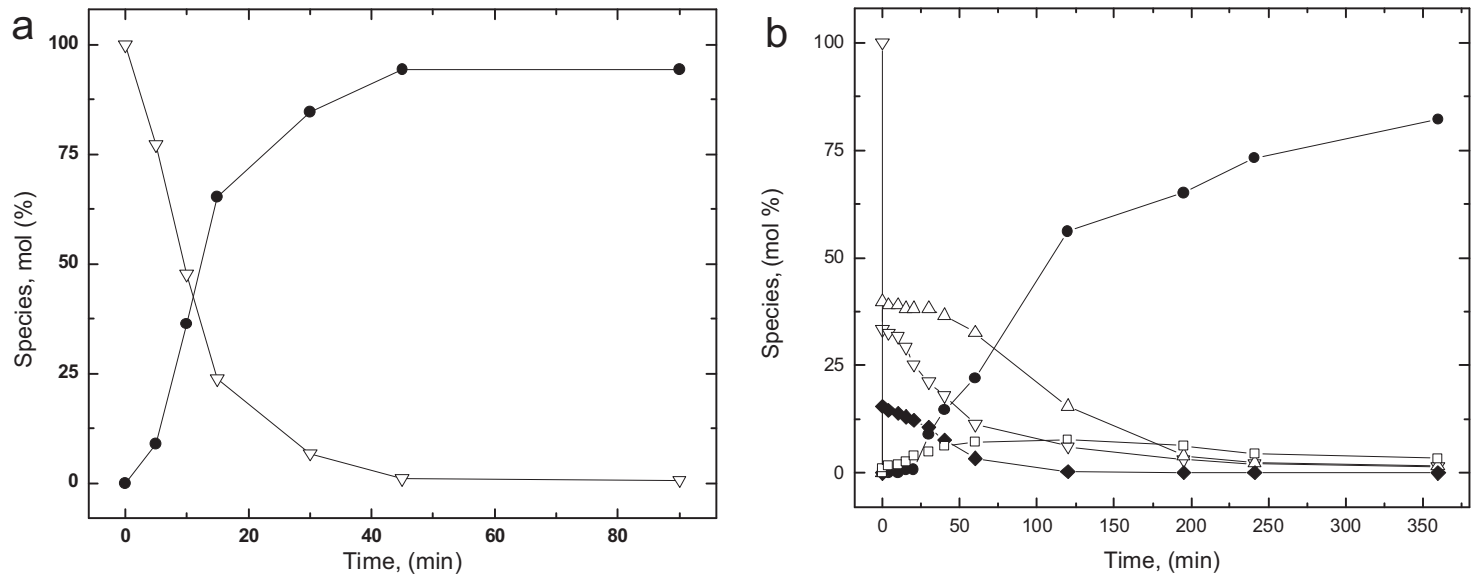

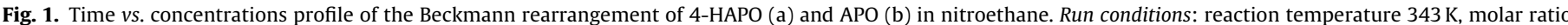

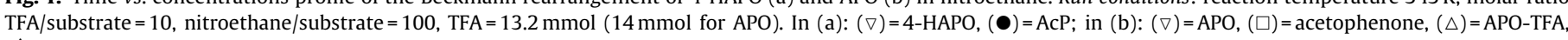
$(\diamond)$ = AcA-TFA, $(\bullet)=$ acetanilide 


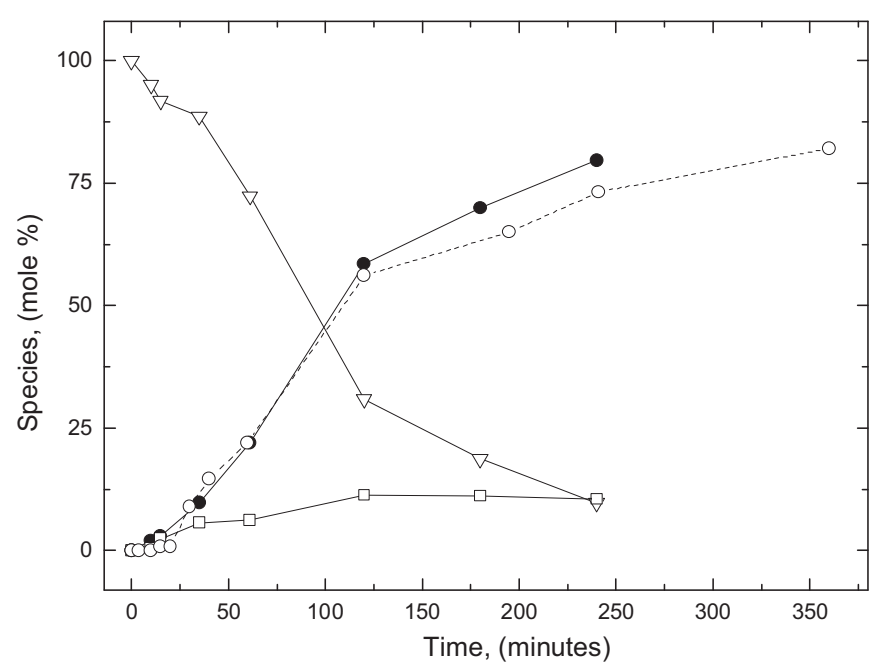

Fig. 2. Time vs. concentrations profile of the Beckmann rearrangement of APO in nitroethane by using GC analysis. Run conditions: reaction temperature $353 \mathrm{~K}$, molar ratio TFA $/$ substrate $=12$, nitroethane $/$ substrate $=100, T F A=14.0 \mathrm{mmol} ;(\nabla)=\mathrm{APO}$, $(\square)=$ acetophenone, $(\bullet)=$ acetanilide, $(\bigcirc)=$ acetanilide GC.

acid catalyzed rearrangement similar to that observed in mineral acid [38-41]. Nevertheless, this marked difference in the reaction path between APO and 4-HAPO, evidenced in Fig. 1 could be only apparent, because of the different method of analysis employed for the two substrates (GC and HPLC, respectively). In fact, the need of using HPLC for analyzing the samples of reaction of 4-HAPO rearrangement may cause the hydrolysis of the intermediates, which, on the contrary, are actually present into the reaction mixture. This is demonstrated by following 4-HAPO rearrangement via NMR in $\mathrm{CDCl}_{3}$, the spectra show complex reaction pattern, where several intermediates are observed, but an unambiguous identification of the intermediates is not possible (see supplementary). A further hint that the lack of the intermediates of the time $v s$. concentrations profile of 4-HAPO rearrangement is due to an analytical problem is evident by noting in Fig. 2 (compare with Fig. 1(b)) that the time $v s$. profile of APO rearrangement is different if the reaction course is followed by HPLC or by GC. As a matter of fact, the various intermediates, evidenced by GC analysis disappear. Fig. 1(b) shows the formation of AcA-TFA, immediately after the addition of TFA thus suggesting a fast rearrangement of APO-TFA.

In order to highlight this behaviors the reactivity of reagents and intermediates with oximes, water and their stability under different conditions are reported in Table 2.

The trifluoroacetylation of both the molecules occurs easily with trifluoroacetic anhydride. In fact, the anhydride reacts with 4-HAPO at $298 \mathrm{~K}$ giving several compounds, whose equilibria after dilution give acetaminophen quantitatively (see supplementary materials). In contrast, APO reacts with trifluoroacetic anhydride giving APO-TFA, which is stable at $298 \mathrm{~K}$, but it rearranges at $343 \mathrm{~K}$ in the presence of TFA. In the gas phase, into the GC injector, APO-TFA rearranges promptly to AcA-TFA (yield 70\%), thus explaining the instantaneous formation of AcP-TFA observed in Fig. 1(b). APO-TFA, AcP-TFA and AcA-TFA are moisture sensitive giving fast hydrolysis to APO and to the corresponding amides and releasing TFA. In the absence of water, AcP-TFA and AcA-TFA react almost quantitatively with APO to APO-TFA and/or to acetanilide and acetaminophen. Furthermore, AcP-TFA and AcA-TFA in the presence of 4-HAPO mediate its rearrangement to acetaminophen also at $298 \mathrm{~K}$ (see Table 2). This explains the trend of Fig. 2, where no intermediates are observed but only APO consumption and acetanilide formation due to the hydrolysis of the intermediates during HPLC analysis. Starting from these results it is clear

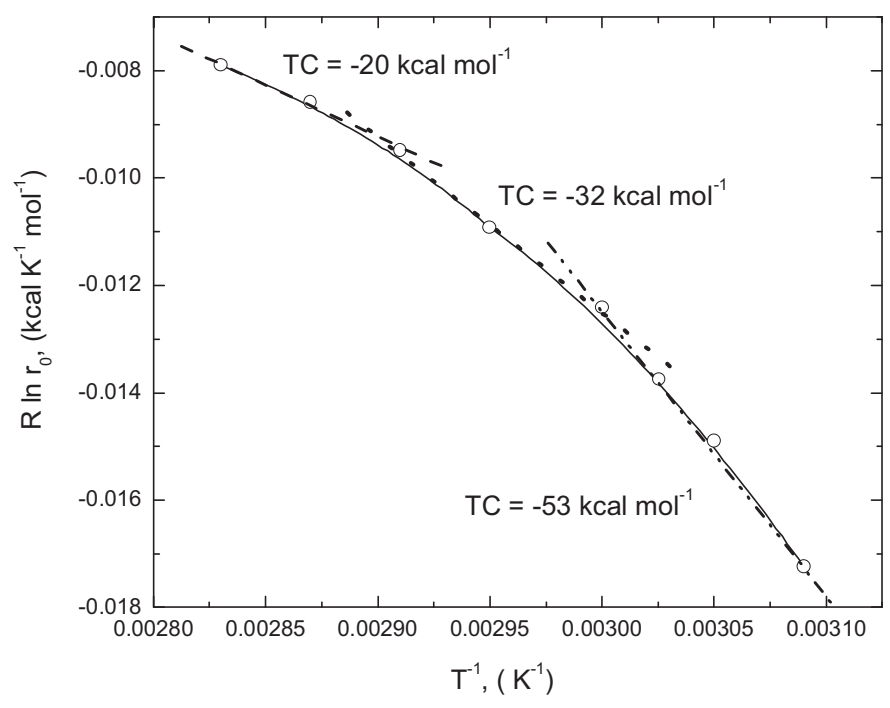

Fig. 3. Arrhenius plot of initial rate from Beckmann rearrangement from 4-HAPO in nitroethane. Run conditions: molar ratio TFA/substrate $=10$, nitroethane $/$ substrate $=100, \mathrm{TFA}=13.2 \mathrm{mmol}$.

that a precise quantification of reaction intermediates cannot be obtained, since thermal rearrangement and hydrolysis alter significantly their distribution vs. time. This behavior suggests that the formation of the trifluoroacetylated-amide intermediate is the key step in the rearrangement of both oximes in agreement with our previous investigation [26-29].

In addition, the reactivity of the trifluoroacetylated amides allows products recovery without work-up operations, which are conversely indispensable in the presence of mineral acids [26-29].

\subsection{Effect of temperature on the initial rate of Beckmann rearrangement in nitroethane as a solvent}

The effect of the temperature on initial rates of rearrangement of 4-HAPO is reported in Fig. 3. The Arrhenius plot of 4-HAPO rearrangement (Fig. 3 ) shows a noticeable non linear trend, thus suggesting a complex reaction path, which is in agreement with the multistep mechanism proposed in the previous section. From an estimate of the temperature coefficient (TC), calculated by dividing in zones the curve of the Arrhenius plot, it appears values of TC ranging from 20 to $50 \mathrm{kcal} \mathrm{mol}^{-1}$. This confirms that several stages influence the overall kinetics, thus the variation of temperature causes the changes of the activation energy of the rate determining step.

At difference of what observed for 4-HAPO, the initial rate of the Beckmann rearrangement of APO is linear (see supplementary materials). Apparently this disagrees with the results of Fig. 1(b), which shows the formation of several intermediates. The value of the TC of about $20 \mathrm{kcal} \mathrm{mol}^{-1}$ is similar to that measured for 4-HAPO at higher temperatures and is practically the same with respect to that measured in mineral acid [38-41].

\subsection{Effect of substrate concentration on the initial rate of Beckmann rearrangement in nitroethane as a solvent}

Fig. 4 reports the influence of the substrate concentration on the initial rate of Beckmann rearrangement of 4-HAPO and APO. As expected in both cases, the initial rate of reaction increases as substrate concentration raise. A power low equation rate satisfactorily fit experimental data because of the linearity of the double logarithmic plot (see supplementary materials) and the calculated 
Table 2

Stability of the reagents and intermediates after $60 \mathrm{~min}$ of reactions under various conditions.

\begin{tabular}{|c|c|c|c|c|c|c|}
\hline \multirow[t]{3}{*}{ Compound tested } & \multicolumn{3}{|c|}{ Stability under various conditions } & \multicolumn{3}{|l|}{ Reagent } \\
\hline & Liquid $298 \mathrm{~K}$ & Liquid $343 \mathrm{~K}$ & Gas GC $533 \mathrm{~K}$ & APO $343 \mathrm{~K}$ & 4-HAPO $343 \mathrm{~K}$ & $\mathrm{H}_{2} \mathrm{O} 298 \mathrm{~K}$ \\
\hline & \multicolumn{6}{|l|}{ Yield \% } \\
\hline 4-HAPO & NR & Est/Re ${ }^{a}$ & $\operatorname{Dec}^{\mathrm{b}}$ & l & 1 & $\mathrm{Hy}^{\mathrm{c}} / \mathrm{Re}=1 / 2$ \\
\hline APO & NR & Est/30 & NR & 1 & 1 & $\mathrm{Hy}^{\mathrm{c}}=1$ \\
\hline 4-HAPO-TFA & $\operatorname{Re}=100$ & $\operatorname{Re}=100$ & $\operatorname{Dec}^{\mathrm{b}}$ & 1 & l & 1 \\
\hline APO-TFA & NR & $\operatorname{Re}=30$ & $\mathrm{Re}=70$ & I & i & $\mathrm{Hy}=100^{\mathrm{d}}$ \\
\hline AcP-TFA & NR & NR & $\operatorname{Dec}^{\mathrm{b}}$ & $\mathrm{Ex} / \mathrm{Re}=100 / 30$ & $E x / \operatorname{Re}=100 / 40$ & $\mathrm{Hy}=100^{\mathrm{e}}$ \\
\hline AcA-TFA & NR & NR & NR & $E x=100$ & $E x / \operatorname{Re}=100 / 40$ & $\mathrm{Hy}=100^{\mathrm{e}}$ \\
\hline
\end{tabular}

Run conditions: solvent $\mathrm{CH}_{3} \mathrm{CN}$, reaction volume $10 \mathrm{~mL}$, TFA concentration $0.1 \mathrm{~mol} \mathrm{~L}^{-1}, \mathrm{TFA} /$ substrate $=1$, substrate $/$ reagent $=1$.

Est $=$ esterification, Gas GC $=$ GC injector, Re = rearrangement, Dec $=$ decomposition, NR = no reaction, Ex = exchange, Hy = hydrolysis .

a Via NMR.

b Traces of trifluoroacetylated compounds are observed but extensive decomposition occurs due to the poor thermal stability of phenols and aminophenols.

c Hydrolysis to ketone.

d Hydrolysis to oxime.

e Hydrolysis to amide

reaction orders are 0.7 and 0.6 for 4 -HAPO and APO, respectively. A non integer reaction order suggests that there are some steps that influence the rate determining step of the reaction $[42,43]$. This is evident for the rearrangement of APO, whose time vs. concentration profile (Fig. 1(b)) shows some intermediates. For instance, the formation of APO-TFA, which is an ester, is a step whose contribution easily explains a non integer reaction order [42,43]. It is likely that also for 4-HAPO rearrangement the kinetics should be influenced by analogous esterification and protonation equilibria, thus explaining the non integer reaction order and the variation of the TC with temperature.

\subsection{Influence of TFA on the initial rate of Beckmann rearrangement in nitroethane as a solvent}

In Table 3 the influence of the TFA/substrate ratio on conversion and selectivity after $18 \mathrm{~h}$ of reaction is reported. It is noteworthy that at a ratio higher than 3 the conversion is practically quantitative and selectivity is as high as $95 \%$ in the desired amide and only at TFA/substrate ratio of 1 ketone formation occurs noticeably. The reason of such a behavior is likely due to the esterification equilibria of the oxime, which is favored at the highest TFA/substrate.

In Fig. 5 the trend of the initial rate of rearrangement of 4-HAPO and APO vs. the concentration of TFA is reported.

In both cases the trend of the initial rate of rearrangement increases with respect to TFA concentration up to $2 \mathrm{~mol} \mathrm{~L}^{-1}$. At TFA concentrations higher than $2 \mathrm{~mol} \mathrm{~L}^{-1}$, the rate of rearrangement of 4-HAPO reaches a plateau and after $2.5 \mathrm{~mol} \mathrm{~L}^{-1}$ also a slight decrease can be noted. Analogous trend is observed also for APO, with a constant increase till $3.2 \mathrm{~mol} \mathrm{~L}^{-1}$, at higher concentration two phases is observed. It is likely that, as already observed for the Beckmann rearrangement of cyclohexanone oxime in TFA- $\mathrm{CH}_{3} \mathrm{CN}$, protonation, esterification and exchange between the trifluoroacetylated amide and the starting oxime, are all reactions affecting the overall reaction rate [26-29].

\subsection{Beckmann rearrangement in TFA as catalyst and solvent}

Fig. 6 shows the time $v s$. concentration profiles regarding to the Beckmann rearrangement of 4-HAPO and APO, respectively, catalyzed by neat TFA, which acts also as the solvent. Both reaction profiles are similar to those observed in the presence of nitroethane as a solvent (see Fig. 1), except the absence of acetophenone as byproduct, for the rearrangement of APO, which causes a further increase of the selectivity toward the acetanilide (see Fig. 6(b)). The same behavior of the reaction carried out in the presence of nitroethane (Fig. 2) is observed by analyzing the samples via HPLC (see supplementary) in which no intermediates are observed for APO rearrangement. This is in agreement with what already discussed in Section 3.2 suggesting that HPLC analysis causes the hydrolysis of the trifluoroacetylated intermediates (4-HAPO-TFA and AcP-TFA) also in the case of 4-HAPO.
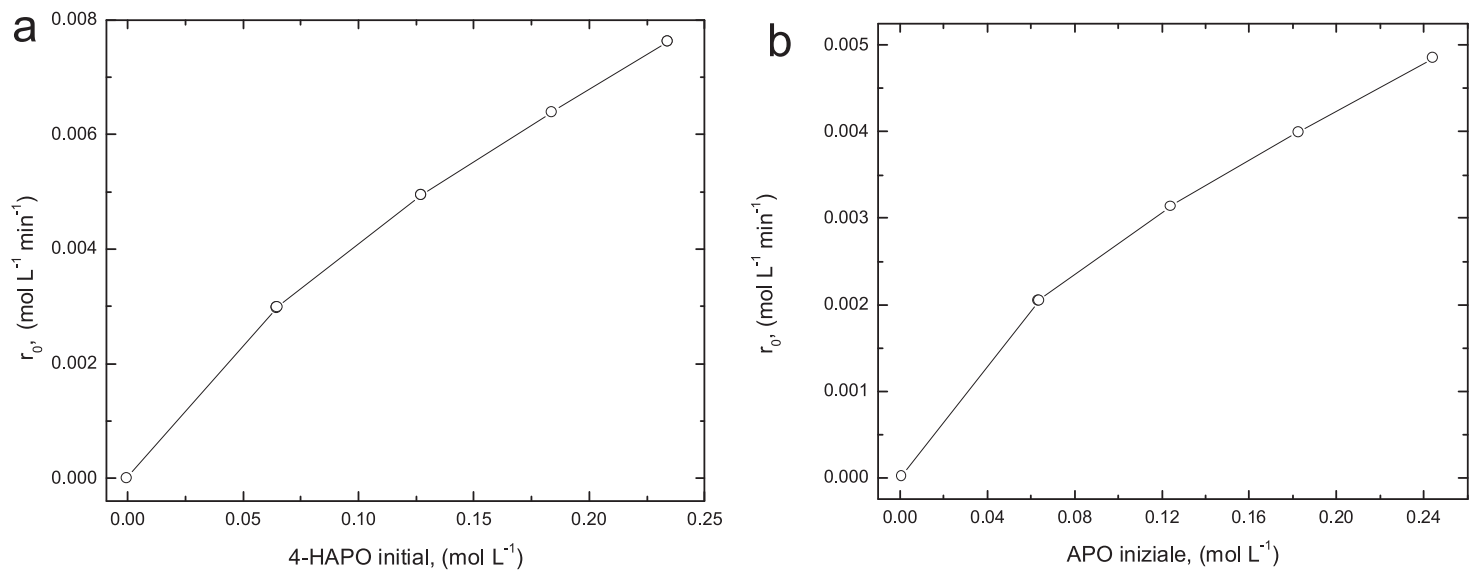

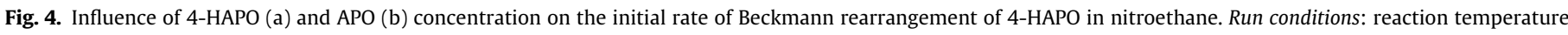
$343 \mathrm{~K}$, molar ratio nitroethane $/ \mathrm{TFA}=10, \mathrm{TFA}=13.2 \mathrm{mmol}(14 \mathrm{mmol}$ for APO $)$. 
Table 3

Influence of the TFA/substrate ratio.

\begin{tabular}{|c|c|c|c|c|c|c|c|c|c|}
\hline \multirow[t]{3}{*}{ Entry } & \multirow[t]{3}{*}{ TFA/substrate } & \multicolumn{4}{|l|}{ 4-HAPO } & \multicolumn{4}{|l|}{ APO } \\
\hline & & \multirow[t]{2}{*}{ Conv. ${ }^{\text {a }}(\%)$} & \multicolumn{3}{|c|}{ Selectivity (\%) } & \multirow[t]{2}{*}{ Conv. ${ }^{\text {a }}(\%)$} & \multicolumn{3}{|c|}{ Selectivity (\%) } \\
\hline & & & Amide & Ketone & Others $^{\mathrm{b}}$ & & Amide & Ketone & Others $^{c}$ \\
\hline 1 & 1 & 64 & 29 & 70 & 1 & 55 & 5 & 75 & 20 \\
\hline 2 & 1.5 & 90 & 51 & 31 & 18 & 84 & 27 & 60 & 13 \\
\hline 3 & 2 & 99 & 76 & 14 & 10 & 91 & 46 & 20 & 14 \\
\hline 4 & 3 & 99 & 95 & 3 & 2 & 99 & 94 & 3 & 3 \\
\hline 5 & 5 & 99 & 98 & 1 & 2 & 99 & 96 & 1 & 3 \\
\hline 6 & 10 & 99 & 99 & 1 & 1 & 99 & 98 & I & 2 \\
\hline
\end{tabular}

Run conditions: solvent $\mathrm{CH}_{3} \mathrm{CH}_{2} \mathrm{NO}_{2}$, reaction volume $10 \mathrm{~mL}$, TFA concentration $0.1 \mathrm{~mol} \mathrm{~L}^{-1}, T=353 \mathrm{~K}$, time of reaction $18 \mathrm{~h}$.

a Oxime conversion.

b Condensation products.

c Others are mainly trifluoroacetylated amide and ketoxime.

\subsection{Effect of temperature on initial rate of Beckmann rearrangement in TFA}

The trend of the Arrhenius plot of the initial rate of rearrangement of 4-HAPO and APO are similar of those obtained in nitroethane (see supplementary materials), with a non constant value of the TC for 4-HAPO comprised between 7 and $30 \mathrm{kcal} \mathrm{mol}^{-1}$. As already pointed out for the reaction carried in the presence of nitroethane as a solvent, this behavior suggests a complex reaction path, where several stages in stationary state are involved. The non homogeneous values of the TC for APO rearrangement are further evidences that the reaction is multistep with equilibria $[42,43]$.

The value of TC for the rearrangement of 4-HAPO in neat TFA is lower than that observed in the presence of nitroethane, suggesting a stabilization effect of the activated state in the presence of neat TFA. The TC for APO rearrangement is practically the same
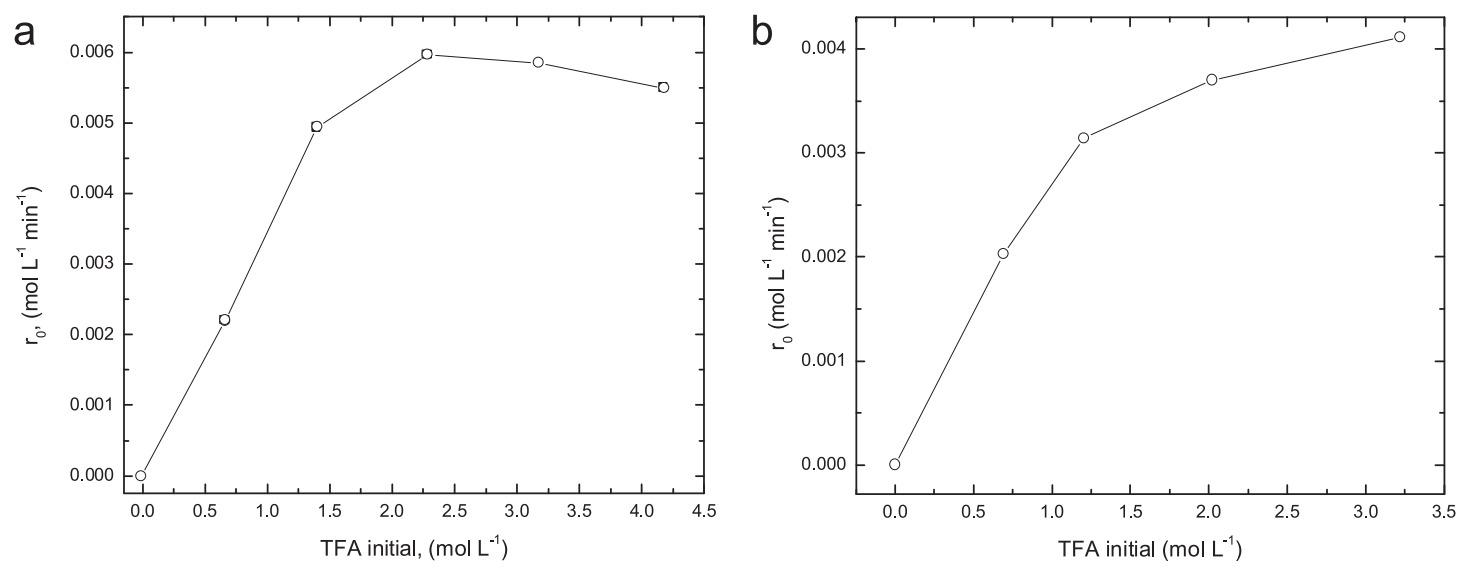

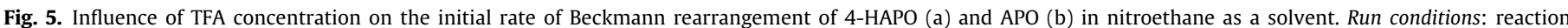
temperature $363 \mathrm{~K}$, molar ratio nitroethane $/ \mathrm{TFA}=10$, nitroethane/substrate $=100, \mathrm{TFA}=13.2 \mathrm{mmol}(14.0 \mathrm{mmol}$ for $\mathrm{APO})$, molar ratio nitroethane $/$ substrate $=100$.
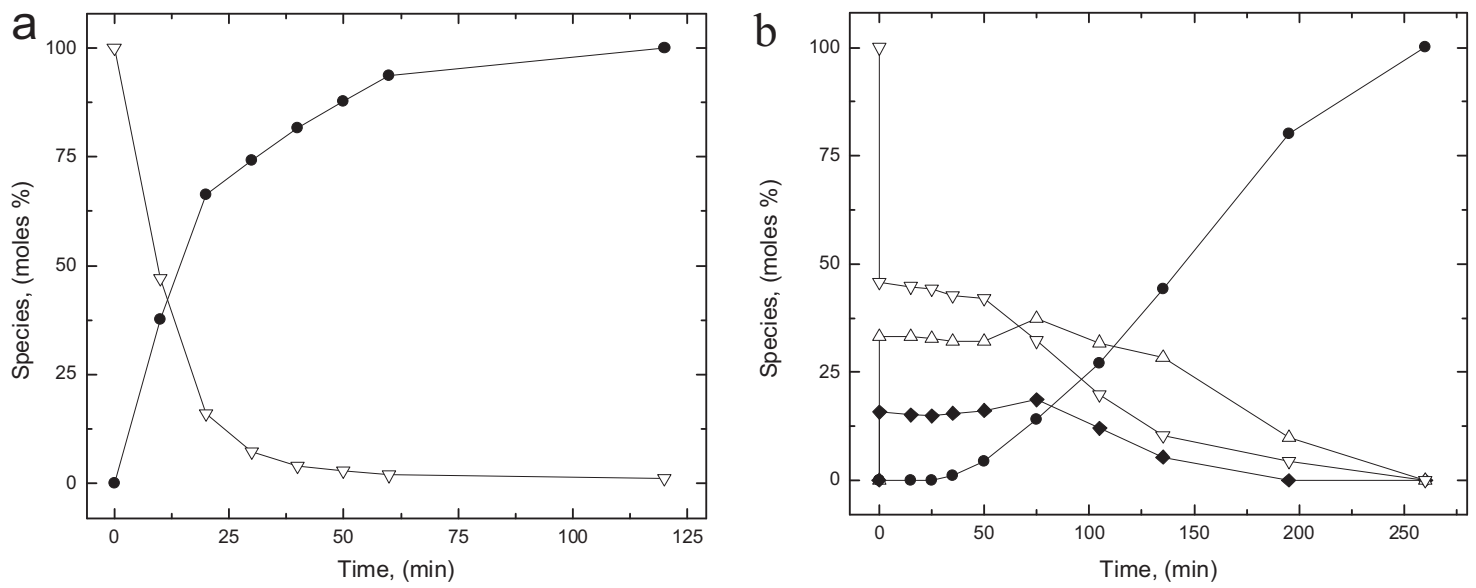

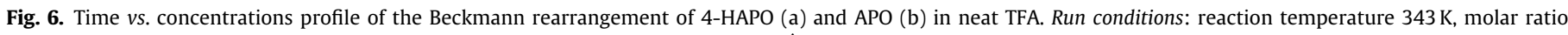
TFA/substrate = 10, TFA = $33.1 \mathrm{mmol}$. In $(\mathrm{a}):(\nabla)=4-\mathrm{HAPO},(\bullet)=\mathrm{AcP}$; in $(\mathrm{b}):(\nabla)=\mathrm{APO},(\bullet)=\mathrm{AcA}-\mathrm{TFA},(\triangle)=\mathrm{APO}-\mathrm{TFA},(\bullet)=\mathrm{acetanilide}$. 
Table 4

Influence of the TFA/substrate ratio.

\begin{tabular}{|c|c|c|c|c|c|c|c|c|c|}
\hline \multirow[t]{3}{*}{ Entry } & \multirow[t]{3}{*}{ TFA/substrate } & \multicolumn{4}{|l|}{ 4-HAPO } & \multicolumn{4}{|l|}{ APO } \\
\hline & & \multirow[t]{2}{*}{ Conv. ${ }^{\mathrm{a}}(\%)$} & \multicolumn{3}{|c|}{ Selectivity (\%) } & \multirow[t]{2}{*}{ Conv. $^{a}(\%)$} & \multicolumn{3}{|c|}{ Selectivity (\%) } \\
\hline & & & Amide & Ketone & Others $^{b}$ & & Amide & Ketone & $\overline{\text { Others }^{\mathrm{c}}}$ \\
\hline 1 & 1 & 66 & 70 & 29 & 1 & 56 & 8 & 16 & 62 \\
\hline 2 & 1.5 & 99 & 85 & 3 & 12 & 99 & 58 & 2 & 40 \\
\hline 3 & 2 & 99 & 94 & 2 & 4 & 99 & 93 & 2 & 5 \\
\hline 4 & 3 & 99 & 96 & 2 & 2 & 99 & 95 & 2 & 2 \\
\hline 5 & 5 & 99 & 98 & 1 & 2 & 99 & 98 & 1 & 2 \\
\hline 6 & 10 & 99 & 99 & 1 & 1 & 99 & 99 & 1 & 1 \\
\hline
\end{tabular}

Run conditions: substrate $1 \mathrm{mmol} T=353 \mathrm{~K}$, time of reaction $18 \mathrm{~h}$.

a Oxime conversion.

b Condensation products.

c Others are mainly trifluoroacetylated amide and ketoxime.

of that measured in the presence of nitroethane as a solvent $\left(\mathrm{TF}=21 \mathrm{kcal} \mathrm{mol}^{-1}\right)$.

\subsection{Effect of substrate concentration on the initial rate of Beckmann rearrangement in TFA}

In Table 4 the influence of the TFA/substrate ratio on conversion and selectivity after $18 \mathrm{~h}$ of reaction is reported. As in the presence of nitroethane as a solvent at a ratio higher than 3 the conversion is practically quantitative and with amides selectivity higher than $95 \%$.

In Fig. 7 are reported the initial rate of 4-HAPO and APO. Fig. 7 shows in both cases an increasing trend of the initial rate of rearrangement as the concentration of substrate raise. As regard 4-HAPO, the power law kinetics gives a good fit of the experimental data with a reaction order of 1.72 (see supplementary materials), at difference of what observed in nitroethane as a solvent, where the kinetics shows a reaction order lower than 1 (0.72). This behavior is likely due to the solvent effect on the equilibria (for instance protonation and/or esterification of oxime) in which the substrate is involved, and not from a change of reaction mechanism for the absence of the solvent $[42,43]$.

Fig. 7(b) reports the trend of initial rate of rearrangement $v$ s. APO initial concentration; the power law kinetics fits the data giving an overall reaction order of about 1.78 , which is also in this case greater than 1 . In this way, the reaction order is increased of an unity compared to that measured in the reaction carried out in nitroethane. Also in this case, a solvent effect is likely acting on the various equilibria present in the reaction environment $[35,42,43]$.

\subsection{Hypothesis on reaction mechanism and reactivity of the intermediates}

The reaction path for APO rearrangement is clearly via the intermediates APO-TFA and AcA-TFA, which are evidenced in the reaction mixture (Figs. 1(b) and 6(b)) and isolated (see supplementary). In additions, the reactivity of the intermediates suggests the active role of TFA as organocatalyst being the essential component in their formation and not only the proton donor. In any case, a reaction path via a purely Brønsted acid catalysis cannot be completely ruled out being the oxime easily protonated in this range of acidity [38,39]. In addition, the FT-IR spectra of the 2,4,6trimethylacetophenone oxime in the presence of TFA at $298 \mathrm{~K}$ do not show formation of nitrilium ion (see spectra in supplementary materials), which is observed in strong mineral acid under the same conditions with this oxime [38-41].

The induction period in the acetanilide formation is a further evidence that a Brønsted acid catalysis for APO Beckmann rearrangement is poorly probable. As a matter of fact, protonation is a fast reaction, and then it does not explain the induction period observed in acetanilide formation. On the contrary, the explanation of an induction period could be connected with the formation of a long living intermediate, which retards the conversion to the final product $[42,43]$. Another possible explanation of the long induction period could be related to an autocatalytic phenomena $[42,43]$. For these reasons it is more likely that the induction period in acetanilide formation is caused by the needs of forming AcA-TFA, which is the intermediate that close the catalytic cycle (Scheme 2). These evidences suggest a reaction mechanism (Scheme 2), which
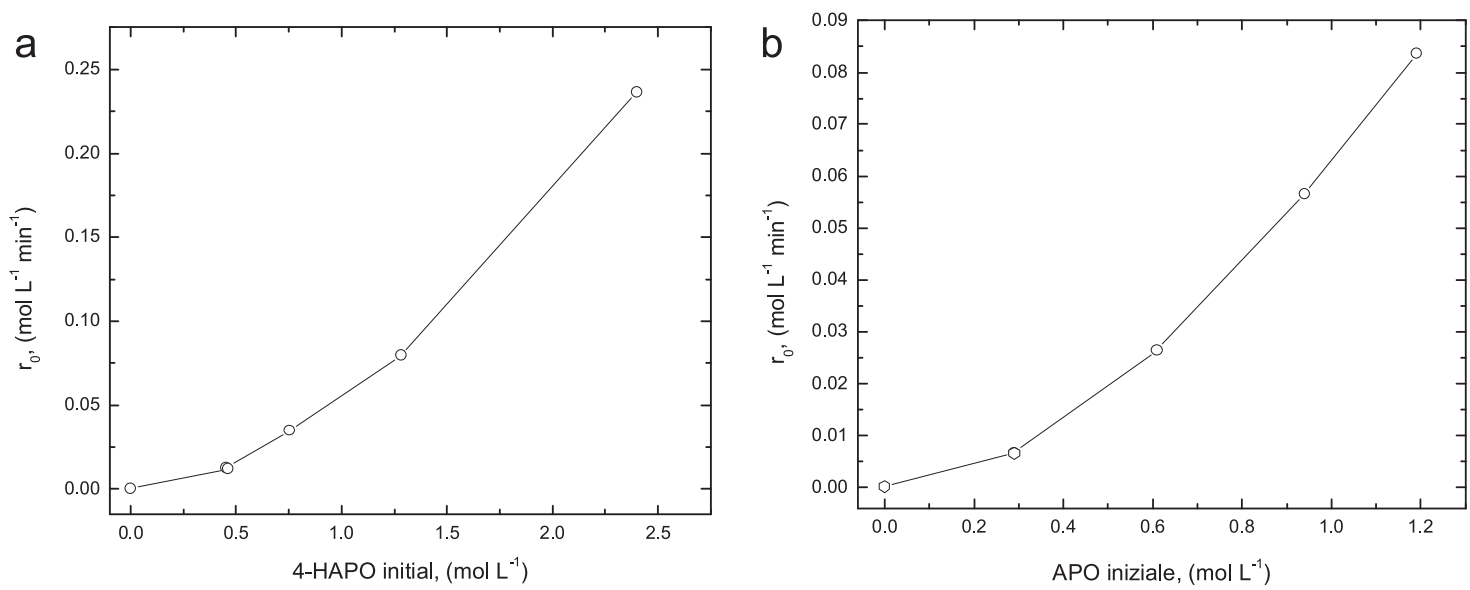

Fig. 7. Influence of 4-HAPO (a) and APO (b) concentration on the initial rate of rearrangement. Run condition: reaction temperature $343 \mathrm{~K}$, TFA=33.1 mmol. 


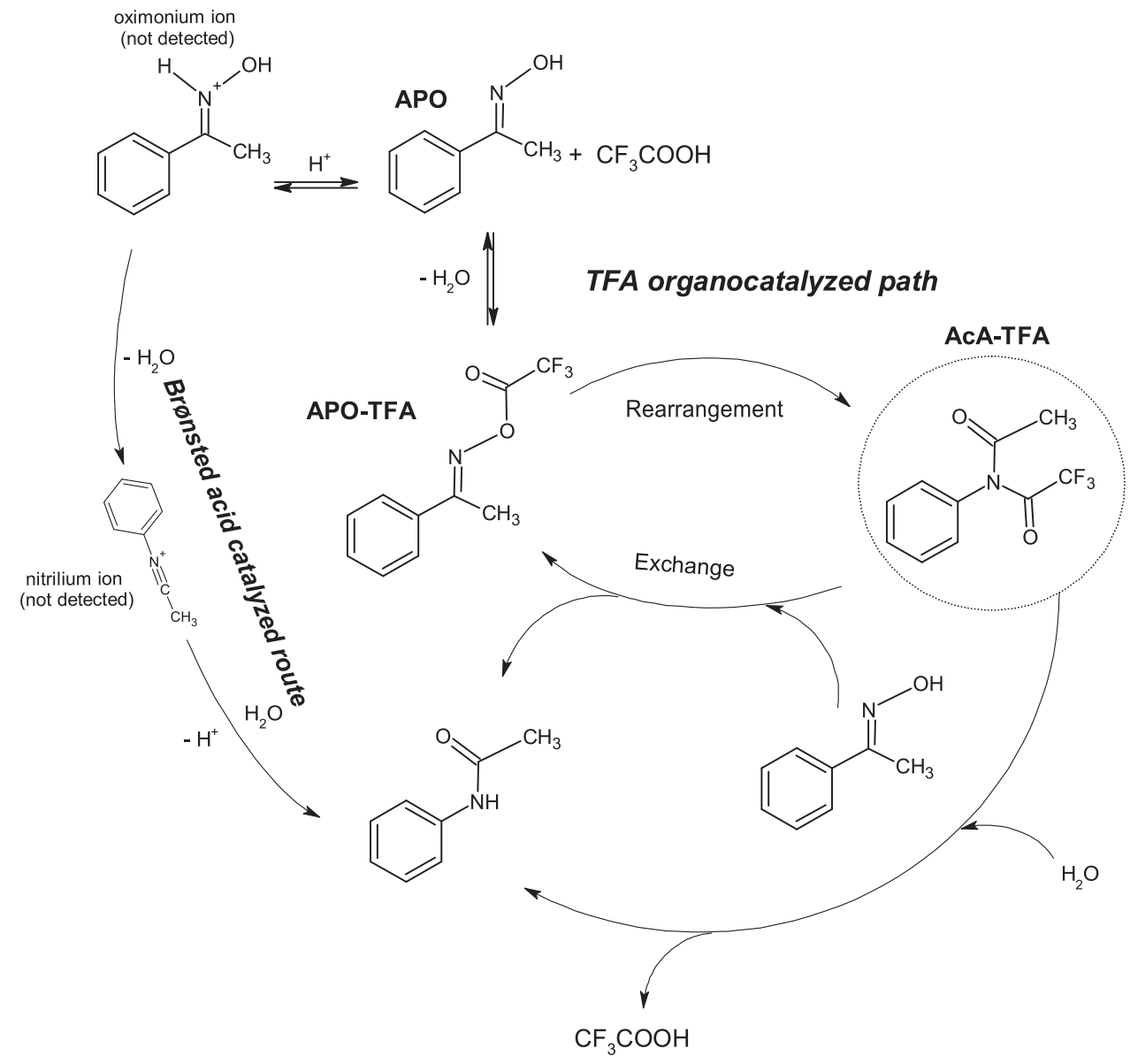

Scheme 2. Mechanism of the Beckmann rearrangement catalyzed by TFA.

is in agreement with that proposed for the cyclohexanone oxime and including the following steps:

(i) APO reacts with TFA to give the ester APO-TFA (equilibrium) [26-29]. This compound has a highly electron deficient nitrogen atom, thus allowing easy Beckmann rearrangement [38-41].

(ii) APO-TFA rearranges to AcA-TFA (irreversible) [38-41].

(iii) AcA-TFA reacts with APO to give acetanilide plus APO-TFA and with water to give TFA plus acetanilide.

Unfortunately, it is not possible a straight parallelism between the reactivity of 4-HAPO with that of APO, since the time vs. concentration profile of the former present apparently no evident intermediates, then it does not allow any direct comparison. However, as already pointed out in Section 3.2, the absence of intermediates is due to their hydrolysis during samples preparation for carrying out HPLC analysis. 4-HAPO rearrangement has been followed by NMR in $\mathrm{CDCl}_{3}$ and it shows complex reaction pattern, where several intermediates are observed, but a sure identification of these intermediates is not possible (see supplementary materials). In addition, the reactivity of APO and 4-HAPO with AcP-TFA gives almost instantly at room temperature exchange reaction to acetanilide plus acetaminophen and a further rearrangement to acetaminophen is observed for 4-HAPO (see Table 2). Starting from these evidences, even though, AcP-TFA has not be directly observed during the reaction, it is likely that AcP-TFA could be involved in 4-HAPO rearrangement, thus suggesting to follow the same mechanism proposed for APO (Scheme 2). Fig. 8 reports some NMR spectra relating the transformation of 4-HAPO in trifluoroacetic anhydride to AcP-TFA.

At difference of APO-TFA, we are not able to isolate 4-HAPOTFA (see Table 2), but the NMR spectra of the reaction of 4-HAPO with trifluoroacetic anhydride at $298 \mathrm{~K}$ in DMSO gives some useful information. For instance (see spectra 3-4), in the presence of 0.3 and 0.7 equiv. of trifluoroacetic anhydride (with respect to the 4-HAPO) it appears two species: AcP-TFA and the second one has the same spectrum of the 4-HAPO in the presence of TFA (compare with spectrum 2). Increasing to 0.9 equiv. of trifluoroacetic anhydride, the signal of the AcP-TFA remains practically the only one (compare with spectra 5 and 6). It is noteworthy to remember that the reaction does not occur in the presence of TFA and in DMSO as a solvent (see Table 1), but is fast in the presence of trifluoroacetic anhydride at $298 \mathrm{~K}$, too. It is likely that a charged intermediate is not necessary, in the presence of trifluoroacetic anhydride, since it is a quite strong neutral electrophile whose reactivity is less influenced by solvent polarity [35]. This behavior is in agreement with the formation of a neutral intermediate such as the ester (for instance APO-TFA and/or 4-HAPO-TFA), which undergoes rearrangement without the involvement of an acid catalysis. The presence of the strongly solvating DMSO or DMC stabilizes the protonated oximes, which are not involved in the catalytic process. The complex behavior of the TC is a clear evidence that the 4-HAPO rearrangement follows a multistep mechanism, since this behavior cannot be explained merely in terms of a Brønsted acid catalysis $[42,43]$. The multistep organocatalyzed reaction mechanism, which is evident for APO, explains without contradictions also the reactivity of 4-HAPO, of ACP-TFA and the complex behavior of the TC, for 


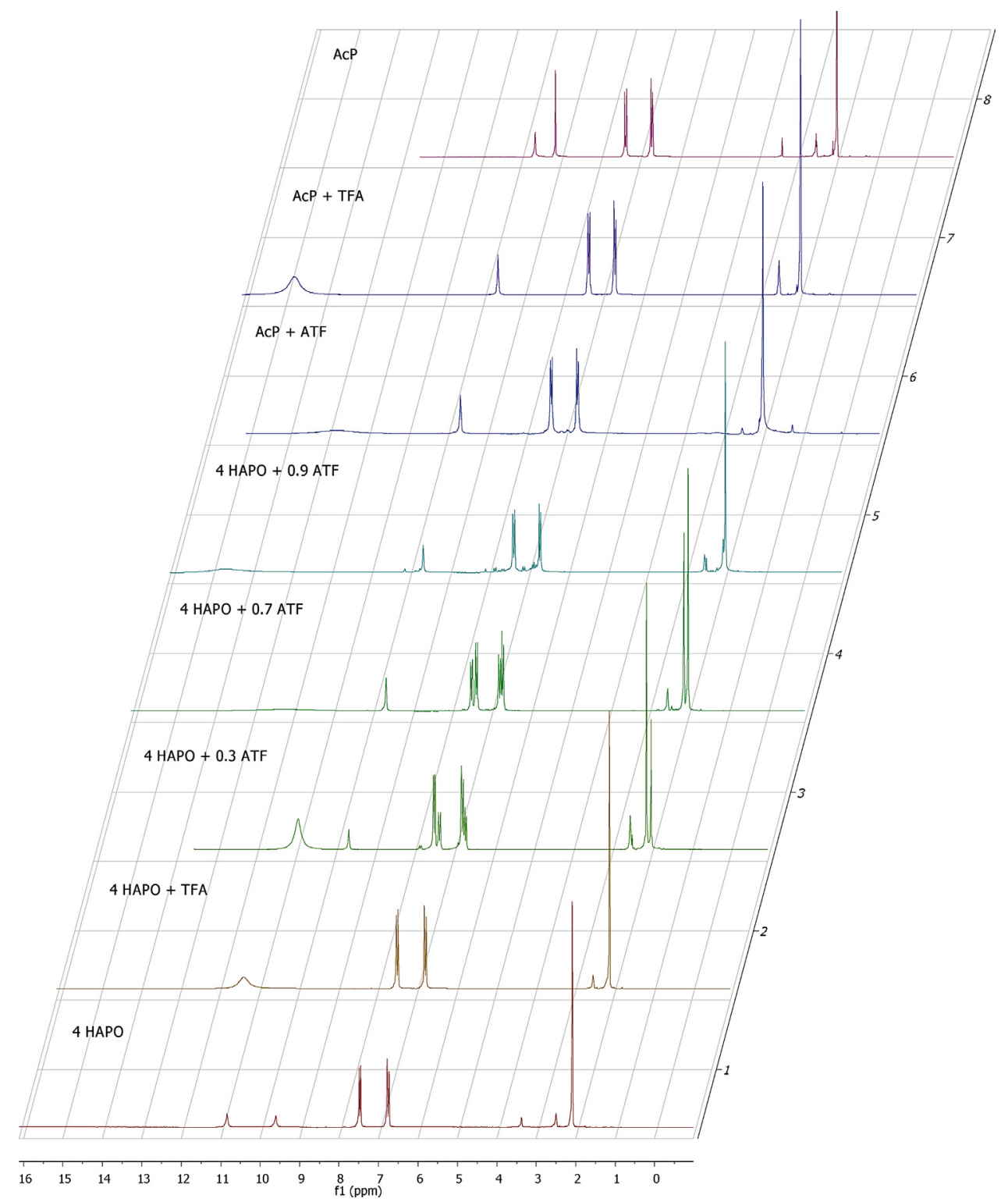

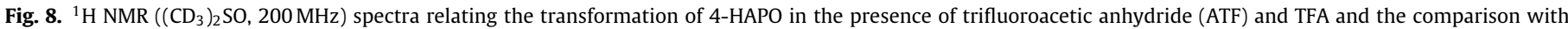
AcP-TFA and acetaminophen (AcP) in the presence of TFA.

this reasons, it appears more likely than the Brønsted acid catalyzed path.

\section{Conclusions}

The use of TFA in the synthesis of paracetamol and acetanilide with respect to the traditional processes which employ mineral acid or thionyl chloride presents the following benefits:

\section{i. lower toxicity;}

ii. easier separation of reaction mixture and recyclable solvent;

iii. TFA may be used pure or in combination with other aqueous or not aqueous solvent.

Besides, the new features of the synthetic procedure, we propose a reaction pathway where TFA acts as an organocatalyst by forming the oxime ester, which rearrange to the corresponding trifluoroacetyl amide, being the latter, in turn, responsible for the trifluoroacetylation of the oxime, closing the catalytic cycle and giving finally the desired amide. This reaction mechanism seems to be quite evident for APO, but by taking into account the behavior of the TC, the reactivity of 4-HAPO in trifluoroacetic anhydride and that of AcP-TFA, such pathway could be reasonably extended also to acetaminophen formation.

\section{Acknowledgements}

Financial support by Ca' Foscari University of Venice is gratefully acknowledged (ADIR fund 2011). A special thank to Mr. Claudio Tortato for the helpful discussions.

\section{Appendix A. Supplementary data}

Supplementary data associated with this article can be found, in the online version, at http://dx.doi.org/10.1016/j.apcata. 2013.12.026. 


\section{References}

[1] M.S.C. Mitchell, R.H. Waring, Aminophenols, Ullmann's Encyclopedia of Industrial Chemistry, sixth ed., Wiley VCH, 1998.

[2] K.G. Davenport, C.B. Hilton, US Patent 4,524,217 A to Celanese Co. (1985).

[3] J.R. Fritch, O.S. Fruchney, T. Horlenko, D.A. Aguilar, C.B. Hilton, P.S. Snyder, W.J. Seelinger, US Patent 5,155,273 to Hechst-Celanese Co. (1992).

[4] http://www.dsir.gov.in/reports/techreps/tsr131.pdf

[5] F.R. Lawrence, W.J. Marshall, Aniline, Ullmann's Encyclopedia of Industrial Chemistry, sixth ed., Wiley VCH, 1998.

[6] G. Petrini, G. Leonfanti, M.A. Mantegazza, F. Pignataro, P.T. Anastas, T.C. Wiliamson, Green Chemistry. Designing Chemistry for the Environment, American Chemical Society, Washington, DC, 1996, pp. 33.

[7] L. Forni, G. Fornasari, F. Trifirò, A. Aloise, A. Katovic, G. Giordano, J.B. Nagy, Microporous Mesoporous Mater. 101 (2007) 153-160.

[8] W.F. Holderich, G. Dahloff, H. Ichihashi, K. Sugita, US Patent 6,531,595 B2 to Sumitomo Chemical Company (2003).

[9] L. De Luca, G. Giacomelli, A. Porcheddu, J. Org. Chem. 67 (2002) 6272-6274.

[10] J.K. Augustine, R. Kumar, A.B. Ashis, B. Mandal, Tetrahedron Lett. 52 (2011) 1074-1077.

[11] L.S. Roselin, R. Selvin, P. Aneesh, M. Bououdina, S. Krishnaswamya, Kinet. Catal. 52 (2011) 823-827.

[12] N.R. Shiju, H.M. Williams, D.R. Brown, Appl. Catal. B: Environ. 90 (2009) $451-457$.

[13] X. Liu, L. Xiao, H. Wua, Z. Li, J. Chen, C. Xia, Catal. Commun. 10 (2009) $424-427$.

[14] Y.M. Chung, H.K. Rhee, J. Mol. Catal. A: Chem. 159 (2000) 389-396.

[15] Y.M. Chung, H.K. Rhee, J. Mol. Catal. A: Chem. 175 (2001) 249-257.

[16] M.J. Climent, A. Corma, S. Iborra, J. Catal. 233 (2005) 308-316.

[17] M. Ghiaci, H. Aghaei, M. Oroojeni, B. Aghabarari, V. Rives, M.A. Vicente, I. Sobrados, J. Sanz, Catal. Commun. 10 (2009) 1486-1492.

[18] A. Zicmanis, S. Katkevica, P. Mekss, Catal. Commun. 10 (2009) 614-619.

[19] K. Ishihara, J. Patent 2006219470 (A) to Nagoya University (2006).

[20] H.P. Krimmer, M. Roos, S. Schauhoff, M. Trageser, Evonik Degussa GmbH EP2013162 A1 (2009).

[21] N. Kaur, P. Sharma, D. Kishore, J. Chem. Pharm. Res. 4 (2012) 1938-1946.
[22] Physical constants of organic compounds, in: D.R. Lide (Ed.), CRC Handbook of Chemistry and Physics, CRC Press, Boca Raton, 2005, p. 3-456.

[23] J.C. Boutonnet, P. Bingham, D. Calamari, C. de Rooij, J. Franklin, T. Kawano, J.-M. Libre, A. McCul-loch, G. Malinverno, J. Martin Odom, G.M. Rusch, K. Smythel, I. Sobolev, R. Thompsonn, J.M. Tiedje, Hum. Ecol. Risk. Assess. 5 (1999) 59-124.

[24] S. His, C. Meyer, J. Cossy, G. Emeric, A. Greine, Tetrahedron Lett. 44 (2003) 8581-8584.

[25] M. Hashimoto, Y. Obora, Y. Ishii, Org. Process Res. Dev. 13 (2009) 411-414.

[26] N.C. Marziano, L. Ronchin, C. Tortato, A. Vavasori, M. Bortoluzzi, J. Mol. Catal. A: Chem. 290 (2008) 79-87.

[27] L. Ronchin, A. Vavasori, M. Bortoluzzi, Catal. Commun. 10 (2008) 251-256.

[28] L. Ronchin, A. Vavasori, J. Mol. Catal. A: Chem. 313 (2009) 22-30.

[29] L. Ronchin, M. Bortoluzzi, A. Vavasori, J. Mol. Struct.: THEOCHEM 858 (2008) 46-50.

[30] J. Zhang, G. Luo, L.V. Yangcheng, K. Wang, CN Patent 102895969 to Tsinghua University (2013).

[31] G. Zhang, X. Wen, Y. Wang, W. Mo, C. Ding, J. Org. Chem. 76 (2011) 4665-4668,

[32] S. Prateeptongkum, I. Jovel, R. Jackstell, N. Vogl, C. Weckbecker, M. Beller, Chem. Commun. (2009) 1990-1992.

[33] L. Liu, J. Hu, X.C. Wang, M.J. Zhong, X.Y. Liu, S.D. Yang, Y.M. Liang, Tetrahedron 68 (2012) 5391-5395.

[34] C.L. Allen, A.R. Chatwal, J.M.J. Williams, Chem. Commun. 48 (2012) 666-668.

[35] C. Reichardt, Solvents and Solvent Effects in Organic Chemistry, second ed., VCH, Weinheim, 1988, pp. 79.

[36] J.H. Smith, J.H. Heidema, E.T. Kaiser, J. Am. Chem. Soc. 94 (1972) 9276-9277.

[37] D.J. Bowden, S.L. Clegg, P. Brimblecombe, Chemosphere 32 (1996) 405-420.

[38] M.I. Vinnik, N.G. Zarakhani, Russ. Chem. Rev. 36 (1967) 51-64.

[39] N.C. Marziano, C. Tortato, L. Ronchin, O. Tonon, R. Bertani, Int. J. Chem. Kinet. 36 (2004) 417-425.

[40] M.T. Nguyen, G. Raspoet, L.G. Vanquickenborne, J. Am. Chem. Soc. 119 (1997) $2552-2562$.

[41] B.J. Gregory, R.B. Moodie, K. Schofield, J. Chem. Soc. B (1970) 338-345.

[42] K.J. Laidler, Chemical Kinetics, second ed., McGraw-Hill, New York, 1965, pp. 321.

[43] J.B. But, Reaction Kinetics and Reactor Design, second ed., Marcel Dekker, NY, 2000, pp. 169. 